\title{
CORONAL ELEMENT ABUNDANCES OF THE POST-COMMON ENVELOPE BINARY V471 TAURI
}

\author{
Martin Still ${ }^{1,2}$ and Gaitee Hussain ${ }^{3}$
}

Evolutionary calculations indicate that there should be no peculiar abundances on the companion star resulting from the common envelope epoch if accretion efficiencies are low. Indeed, we find no evidence for peculiar abundances from archival ASCA data, although uncertainties are high. We find that a singletemperature plasma model does not fit the data. Two-temperature models with decoupled elemental abundances suggest that $\mathrm{Fe}$ is underabundant compared to the Hyades photospheric mean. In the absence of a measurement of photospheric Ne abundance in the cluster, we find Ne is overabundant compared to the solar photospheric value. This is indicative of the inverse first ionization potential effect. Differences between coronal and photospheric abundances are believed to result from the fractionation of ionized and neutral material in the upper atmosphere of the star. Care must be taken to include this effect when inferring photospheric abundances from X-ray data (e.g., Sarna 2004).

V.171 Thu is a 12.5 hom eclipsing binary with a white dwarf and tidally-locked $\mathrm{K} 2$ companion. At a distance of $47 \mathrm{fr}$, it is the closest object which has bern through a recent common envelope phase of erolution. During the red giant phase of the white dwate pregenitor's life, the envelope of the giant was linge enomgh to contain the orbit of the $k$ star companion. While the two stars shared a common envelope, tides, friction and mass loss created a significantly smaller binary, with a short orbital period. The envelope of the giant has since been ejected, but the binary still loses angular moment um through wind braking and gravitational radiation. V471 Tau is therefore one of the best candidates for a pre(ataclysmic vaiable (pre-CV). CVs occur when the orbital period of a white dwarf red dwarf binary becomes short enough for the main-sequence companion to fill it is Roche lobe. Quasi-persistent accretion will then occur through Roche hobe orerflow. These

\footnotetext{
INASA/Goddard Space Flight Center. Cireenbelt. MD 2077. (S.) (Martin.stillugste.nasa.gor).

2 Iniversities Space Research Association.

${ }^{3}$ ESTEC ESA. 2200 AG. Noordwijk. The Netherlands.
}

objects are the sources of dwarf nova outbursts and classical nova eruptions that enrich the galactic ISM.

V471 Tau is a member of the Hyades open cluster. Photospheric abundances of the Hyades members have been measured by Cayrel et al. (1985) and Varenne \& Monier (1999). Accepting model assumptions we show in Still \& Hussain (2003) that coronal $\mathrm{Fe}$ is under-abundant relative to the mean photospheric Hyades content and coronal Ne is overabundant, at least relative to the solar photosphere. The Ne/Fe ratio in V471 Tau is suggestive of an inverse-FIP (First Ionization Potential) effect.

The coronal, energetic particle and cosmic ray abundances in the sun are all different from the solar photospheric content. Mounting evidence indicates that this is equally true for active stars (e.g. Brinkman et al. 2001). While in most cases the photospheric abundances remain uncertain, there is at least one strong piece of evidence for fractionation regions in between stellar photospheres and coronae. It derives from the fact that coronal abundances are correlated with elemental FIP. In the solar atmosphere the ratio of coronal to photospheric abundances decreases with FIP. When comparing stellar coronal abundances to the solar photosphere some sources follow this trend while others show the "inverse FIP effect", i.e. an increasing ratio with increasing FIP.

FIP effects are thought to be directly associated with the process of element fractionation in stellar atmospheres (Hénoux 1995). The ionized fraction of each element will depend on the plasma temperature in the fractionation region. Some process is required to decouple the ionized and neutral plasmas. Possibly the charged particles are accelerated along field lines in loops above the stellar photosphere. Flare ejection provides another mechanism to propel material into the corona.

\section{REFERENCES}

Brinkman, A.C. et al., 2001, A\&A, 365, L324

Cayrel, R.. Cayrel de Strobel, G., Campbell, B., 1985, A\&A, 146,249

Hénoux, J.-C., 1995, Adv. Space Res., 15, (7)23

Sarna, M.. 2004, this volume

Still. M.. Hussain, G., 2003, ApJ, 597, 1059

laremne. O., Monier. R.. 1999. A\&A, 351. 247 